\title{
Influência da Técnica de Anestesia no Tempo de Ocupação de Sala Cirúrgica nas Operações Anorretais
}

\author{
Influence of the Anesthetic Technique on the Time Spent in Operating \\ Rooms in Anorectal Procedures
}

\author{
PAULO GUSTAVO KOTZE - TSBCP'; ELIZABETH MILLATAMBARA²; LUIZCARLOS VON BAHTEN; FÁBIO \\ SILVEIRA ${ }^{4} ;$ EDUARDO WIETZIKOSKI $^{5}$
}

\begin{abstract}
${ }^{1}$ Chefe do Serviço de Coloproctologia do Hospital Universitário Cajuru - PUCPR; ${ }^{2}$ Chefe do Serviço de Anestesiologia da Santa casa de Misericórdia de Curitiba - PUCPR; ${ }^{3}$ Chefe do Serviço de Cirurgia Geral do Hospital Universitário Cajuru - PUCPR; ${ }^{4}$ Médico do Serviço de Cirurgia Geral do Hospital Universitário Cajuru-PUCPR; ${ }^{5}$ Ex-residende do Serviço de Cirurgia Geral do Hospital Universitário Cajuru - PUCPR.
\end{abstract}

KOTZE PG; TAMBARA EM; VON BAHTEN LC; SILVEIRA F; WIETZIKOSKI E. Influência da Técnica de Anestesia no Tempo de Ocupação de Sala Cirúrgica nas Operações Anorretais. Rev bras Coloproct, 2008;28(2): 227-233.

RESUMO: Introdução: atualmente cerca de $90 \%$ das operações anorretais são realizadas em regime ambulatorial. A técnica anestésica é fator fundamental na busca de reduzido tempo de internação, agilidade no ambiente cirúrgico e redução de custos nestes procedimentos. Não há consenso na literatura sobre qual o melhor tipo de anestesia para essas operações. Objetivo: comparar o tempo de ocupação de sala cirúrgica em pacientes submetidos a operações anorretais através da técnica de raquianestesia com bupivacaína $0,5 \%$ isobárica comparada com a técnica de anestesia venosa com propofol associada ao bloqueio perianal local com lidocaína a 2\% e bupivacaína 0,5\%. Métodos: Foram incluídos 99 pacientes divididos em 2 grupos: grupo I (raquianestesia), composto por 50 pacientes e grupo II (anestesia combinada), composto por 49 pacientes. Foram estudados os procedimentos cirúrgicos e o tempo de procedimento anestésico-cirúrgico, e medida indireta da ocupação da sala cirúrgica. Resultados: Não houve diferença estatística significativa entre os grupos estudados em relação ao tipo de procedimento cirúrgico, sexo e idade. $O$ tempo médio do procedimento anestésico-cirúrgico, no grupo I foi de 53,1 min e de 44,08 min no grupo II (p=0,034). Conclusões: As duas técnicas estudadas foram eficazes. Houve menor tempo de procedimento anestésico-cirúrgico nos pacientes operados com anestesia combinada, com significância estatística.

Descritores: Raquianestesia; Propofol; Procedimentos Cirúrgicos Ambulatoriais; Canal Anal; Cirurgia Colorretal.

\section{INTRODUÇÃO}

Na maioria dos grandes centros, o tratamento cirúrgico das doenças anorretais é realizado com o conceito de paciente dia, muito comumente referidas como cirurgias ambulatoriais ${ }^{1}$. A escolha da técnica de anestesia para tais procedimentos é de fundamental importância. Uma técnica anestésica ideal visa boas condições operatórias, agilidade no ambiente cirúrgico, ausência de efeitos colaterais, rápida recuperação com possibilidade de alta precoce e baixo custo ${ }^{2}$. Vá- rios são os estudos publicados na literatura comparando diferentes técnicas anestésicas, demonstrando as vantagens e as desvantagens de cada uma ${ }^{1-3}$.

Quatro técnicas podem ser utilizadas: bloqueio regional espinhal (raquianestesia ou anestesia peridural), anestesia local, anestesia combinada (venosa + local) e anestesia geral. Nenhuma delas é considerada ideal, ou descrita como o padrão-ouro. A experiência do cirurgião, a posição do paciente na mesa cirúrgica, a experiência do anestesiologista e as condições do local são os fatores determinantes para a escolha de uma

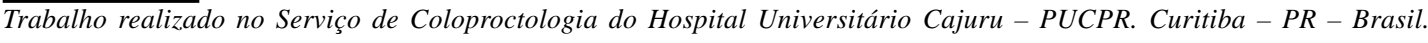

Recebido em 13/02/2008

Aceito para publicação em 29/04/2008 
destas técnicas ${ }^{3}$. Em suma, ainda se busca uma técnica anestésica ideal para o tratamento cirúrgico das doenças anorretais, e estudos sobre o tema são constantemente publicados ${ }^{1-3}$.

Uma das preocupações em relação ao tipo de anestesia para estas operações é o tempo de ocupação da sala cirúrgica, medido indiretamente pelo tempo de procedimento anestésico-cirúrgico. Operações que possam ser realizadas em menor tempo, sem alteração dos resultados finais, facilitam o trabalho no ambiente cirúrgico e podem disponibilizar salas cirúrgicas para outros procedimentos.

\section{OBJETIVO}

O objetivo do presente estudo é o de comparar o tempo de ocupação da sala cirúrgica em pacientes submetidos a operações anorretais através da técnica de raquianestesia com bupivacaína $0,5 \%$ isobárica comparada com a técnica de anestesia venosa com propofol associada ao bloqueio perianal local com lidocaína a $2 \%$ e bupivacaína $0,5 \%$ (anestesia combinada).

\section{MÉTODO}

Este estudo foi realizado no Hospital Universitário Cajuru, no setor de hospital-dia, onde as operações foram realizadas. Após assinatura do termo de consentimento informado, de acordo com as normas locais, os pacientes operados passaram a ser incluídos no banco de dados do Serviço de Coloproctologia do referido hospital, entre janeiro de 2004 e março de 2005.

O projeto de pesquisa foi aprovado pelo Comitê de Ética em Pesquisa da PUCPR (CEP - PUCPR), sob o parecer número 241/07 e registro de número 1867 , permitindo a análise das informações dos pacientes que compuseram o referido banco de dados do serviço.

Os critérios de inclusão dos pacientes para o estudo foram: idade entre 16 e 80 anos, portadores de qualquer doença benigna anorretal com indicação cirúrgica; avaliação anestésica pré-operatória ASA I ou II; condição de compreensão do termo de consentimento informado; presença de acompanhantes e pacientes do sistema público de saúde.

Os critérios de exclusão para o estudo foram: idade menor que 16 anos ou maior que 80 anos; avaliação anestésica pré-operatória ASA III ou IV; concomitância de distúrbios mentais ou psiquiátricos documentados que os impedissem de compreender e concordar com o termo de consentimento informado; ausência de acompanhantes; doenças anorretais com infecção aguda e pacientes portadores de planos de saúde e particulares.

Todas as operações foram realizadas na sala 1 do setor de hospital-dia, mediante as mesmas condições de ambiente. Os atos anestésicos foram realizados sempre pelos mesmos dois anestesiologistas do grupo de anestesiologia do referido hospital, e seguiram padronização técnica minuciosa.

A técnica anestésica foi determinada aleatoriamente antes dos pacientes entrarem no centro cirúrgico. Os mesmos foram encaminhados à sala de número 1, colocados em posição de decúbito-dorsal sobre a mesa cirúrgica e monitorizados. Após a monitorização, foi feita punção venosa periférica, sempre no antebraço esquerdo. A partir deste ponto iniciou-se a cronometragem do procedimento anestésico-cirúrgico. Passou-se então para a anestesia propriamente dita. Não se realizou antibioticoprofilaxia venosa nos pacientes incluídos no estudo.

Os pacientes do grupo I (raquianestesia) foram colocados na posição sentada, com ângulo de 90 graus entre a maca e sua coluna vertebral, em posição para facilitar a introdução da agulha no espaço intervertebral. Após antissepsia marcou-se o espaço L2 - L3, e uma anestesia local com lidocaína $1 \%$, sem vasoconstritor (Xylestesin ${ }^{\circledR}$, Cristália, Brasil) foi realizada na pele e planos adjacentes, com $5 \mathrm{ml}$ de solução. Posteriormente, realizou-se a punção no referido espaço através da área da pele anestesiada, até a penetração no espaço subaracnóideo, com agulha especial para raquianestesia descartável 27 G, com 88 x 0,42 mm (Spinocan ${ }^{\circledR}$, Laboratórios B. Braun S.A.). Após extravasamento de pequena quantidade de líquido cérebro-espinhal, injetou-se solução de $10 \mathrm{mg}$ de bupivacaína $0,5 \%$ isobárica, $2 \mathrm{ml}$, (Cristália, Brasil) no referido espaço. Logo após, os pacientes foram colocados em posição de litotomia, com fixação dos membros inferiores nas perneiras cirúrgicas com ataduras de crepe. Após o teste de sensibilidade, iniciou-se o ato cirúrgico proctológico.

Os pacientes do grupo II (propofol venoso e bloqueio perianal local - anestesia combinada), após a monitorização e a punção venosa, foram sedados pelo anestesiologista com infusão de $2 \mathrm{mg} / \mathrm{kg}$ de propofol (Propovan ${ }^{\circledR}$, Cristália, Brasil). Após a sedação inicial, os pacientes receberam quatro litros por minuto de oxigênio através de máscara facial. Posteriormente, foram colocados na posição de litotomia, com fixação 
dos membros inferiores de maneira idêntica a do grupo I. Procedeu-se à antissepsia da região anal com solução de iodopovidona tópica na quantidade de $20 \mathrm{ml}$, e posterior colocação dos campos estéreis. A solução anestésica local foi então preparada, com utilização de uma seringa de $20 \mathrm{ml}$ e uma agulha de $40 \times 1,2 \mathrm{~mm}$ para aspiração de $10 \mathrm{ml}$ de lidocaína a $1 \%$, sem vasoconstritor, associados a $10 \mathrm{ml}$ de bupivacaína $0,5 \%$. O bloqueio anestésico perianal local foi realizado sempre pelo mesmo cirurgião, que foi responsável por todas as operações dos pacientes incluídos no estudo, com injeção da solução previamente descrita, com agulha $25 \times 0,6 \mathrm{~mm}$ pela técnica descrita por Schneider, em $1950^{4}$ (anestesia subcutânea perianal em leque, superficial, sem hialuronidase). A manutenção da anestesia foi realizada com infusão de $100 \mathrm{ìg} / \mathrm{kg} / \mathrm{minu}-$ to de propofol até o final do ato operatório.

O término da cronometragem do tempo de procedimento anestésico cirúrgico foi marcado ao final da confecção do curativo perianal, nos dois grupos.

Após o término da operação anorretal, os pacientes dos dois grupos foram mantidos em observação na sala de recuperação pós-anestésica imediata, com monitorização da oximetria de pulso. A alta do centro cirúrgico pela equipe de anestesiologia foi efetivada após estabilização.

\section{ANÁLISE ESTATÍSTICA}

As variáveis sexo e procedimentos cirúrgicos realizados (hemorroidectomias, fistulotomias $1 .^{\circ}$ tempo, fistulotomias 2. ${ }^{\circ}$ tempo, fissurectomias anais, ressecção de condilomas e de plicomas), por serem dicotômicas categóricas, foram analisadas inicialmente por teste de qui-quadrado. Para as variáveis idade e tempo de procedimento anestésico-cirúrgico, por serem as mesmas quantitativas contínuas, com pressuposto de normalidade atendido, foi aplicado teste $t$ de student. O nível de significância (p) estabelecido foi de 5\%.

\section{RESULTADOS}

Foram operados 106 pacientes, por procedimentos anorretais, no período entre janeiro de 2004 e fevereiro de 2005 no Serviço de Coloproctologia do Hospital Universitário Cajuru. Destes, 99 pacientes foram incluídos no estudo. Dos 7 pacientes excluídos, 2 eram menores de 16 anos de idade e 5 apresentavam estado-físico pré-operatório ASA III.

Dos 99 pacientes incluídos no estudo, após a escolha aleatória, 50 foram operados com raquianestesia, e compuseram o grupo I. Os 49 pacientes restantes compuseram o grupo II, e foram operados com a anestesia combinada (anestesia venosa com propofol associada ao bloqueio perianal local).

Dos 50 pacientes do grupo I (raquianestesia), 28 eram do sexo feminino e 22 do masculino. A idade destes pacientes variou entre 17 e 71 anos (média de 44,2 anos, com desvio padrão de 12,31 anos). Dos 49 pacientes do grupo II, 25 eram do sexo feminino e 24 do masculino. A idade dos pacientes deste grupo variou entre 17 e 80 anos (idade média de 39,31 anos com desvio padrão de 13,72 anos). Os valores do nível de significância (p) encontrados nesta análise foram de 0,619 para a variável sexo e 0,075 para a idade. Não houve diferença estatística entre os grupos estudados em relação a estas variáveis (tabela 1).

Com relação aos procedimentos cirúrgicos, no grupo I, foram realizadas 32 hemorroidectomias (associação com fissurectomia anal em 5 casos), 9 fistulotomias em 1 tempo, 6 fissurectomias anais com esfincterotomia interna, 2 fistulotomias em 2 tempos (segundo tempo - retirada do reparo esfincteriano e abertura do trajeto) e uma ressecção de plicomas anais de grandes dimensões (gráfico 1). No grupo II, foram realizadas 29 hemorroidectomias (associação com fissurectomia anal em 3 casos), 9 fistulotomias anais em 1 tempo, 4 fistulotomias em 2 tempos, 4 fissurectomias anais com esfincterotomia interna e 3

Tabela 1 - Resultados da análise estatística da idade, sexo e procedimentos realizados, $p<0,05$.

\begin{tabular}{lclc}
\hline Variável analisada & Valor de $\mathbf{p}$ & Variável analisada & Valor de p \\
\hline Idade & 0,075 & Fístulas 2 tempos & 0,385 \\
Sexo & 0,619 & Fissurectomias & 0,451 \\
Hemorroidectomia & 0,622 & Condilomas & 0,075 \\
Fístulas 1 tempo & 0,962 & Plicomas & 0,319 \\
\hline
\end{tabular}


ressecções de condilomas anais. Não houve ressecção de plicomas anais neste grupo (gráfico 2). Não houve diferença significante em nenhum dos procedimentos cirúrgicos realizados, analisados isoladamente entre os grupos. Portanto, permite-se afirmar que não houve diferença entre os grupos estudados na indicação cirúrgica (tabela 1).

O tempo do procedimento anestésico-cirúrgico, no grupo I, variou entre 20 e 120 minutos (média de 53,1 minutos e desvio padrão de 22,2 minutos). No grupo II, houve variação entre 10 e 120 minutos (média de 44,08 minutos e desvio padrão de 19,38 minutos). O nível de significância encontrado (p) foi de 0,034 . Estes resultados estão sumarizados na tabela 2. Houve diferença significante entre os dois grupos, e permitese afirmar que os pacientes do grupo II tiveram menor tempo de procedimento quando comparados com o grupo I (gráfico 3).

\section{DISCUSSÃO}

Cerca de 5\% da população adulta americana é portadora de doenças anorretais 5 . Em torno de 10\% destes pacientes apresentam necessidade de tratamento cirúrgico para melhora de suas queixas ${ }^{6}$.

A escolha da anestesia para operações sobre o reto e o ânus é fundamental para o sucesso das mesmas. Não há consenso na literatura sobre qual a melhor técnica anestésica para este fim². Todas as técnicas descritas apresentam vantagens e desvantagens ${ }^{1}$. Poucos trabalhos são conclusivos em determinar qual delas é a melhor. Fleischer et al., em 1994, demonstraram que a anestesia local é superior à anestesia epidural em operações proctológicas, por menores complicações e custos?

Atualmente, cerca de $90 \%$ dos pacientes portadores de doenças anorretais podem ser operados com o conceito de paciente-dia ${ }^{2}$. Escolher uma técnica anestésica que possibilite esta condição, com baixos

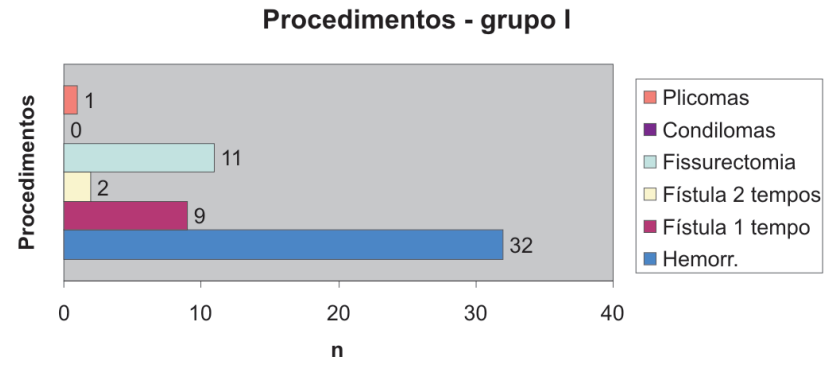

Gráfico 1 - Procedimentos cirúrgicos realizados - grupo I.

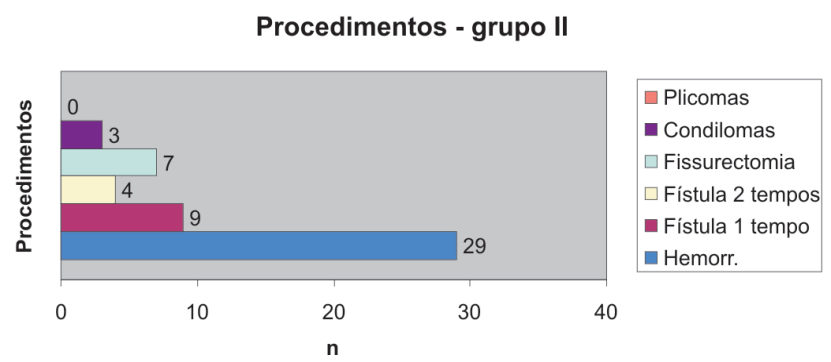

Gráfico 2 - Procedimentos cirúrgicos realizados - grupo II.

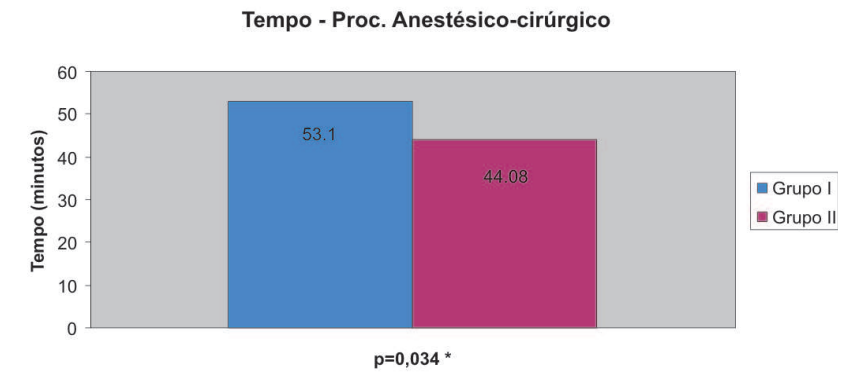

Gráfico 3 - Tempo de procedimento anestésico-cirúrgico nos grupos I e II.

custos e com boa eficácia, com conforto para os pacientes e equipe cirúrgica, é objeto de diversos estudos na literatura mundial ${ }^{2,3,8,9}$. Há serviços que utilizam como primeira opção a anestesia venosa associada ao bloqueio local ${ }^{8}$. Outros descrevem que procedimentos ambulatoriais podem também ser realizados com bloqueio epidural, conforme trabalho nacional de Imbelloni

Tabela 2 - Resultados do tempo de procedimento anestésico-cirúrgico sumarizados com análise estatística (teste t de student com nível de significância de 5\% - p<0,05).

\begin{tabular}{lccccccccc}
\hline & \multicolumn{2}{c}{ Média } & \multicolumn{2}{c}{ Desvio-padrão } & \multicolumn{2}{c}{ Mínimo } & \multicolumn{2}{c}{ Máximo } & Valor de p \\
\cline { 2 - 8 } Grupo & I & II & I & II & I & II & I & II & \\
\hline $\begin{array}{l}\text { Tempo Proc. Anestésico- } \\
\text { cirúrgico (minutos) }\end{array}$ & 53,10 & 44,08 & 22,20 & 19,38 & 20 & 10 & 120 & 120 & $0,034 *$ \\
\hline
\end{tabular}


et al., publicado em $2004^{10}$. A ampla discussão sobre qual a mais adequada técnica de anestesia para operações proctológicas motivou a realização desta análise.

Foi criado no Hospital Universitário Cajuru o setor de hospital-dia, centro cirúrgico específico para procedimentos de pequeno porte . Logo nos primeiros meses de trabalho neste setor, pôde-se perceber uma maior agilidade e menor tempo de ocupação das salas cirúrgicas, subjetivamente. Com isto, pôde-se realizar um número maior de operações nos determinados períodos, aproveitando-se mais o tempo e as atividades de ensino para acadêmicos e residentes. Uma grande característica dos pacientes operados neste setor era a rápida alta hospitalar, sem perda da eficácia dos procedimentos.

Em suma, um paciente operado ambulatorialmente pôde ocupar durante menor tempo as salas cirúrgicas, não ocupou leitos hospitalares de internação e possivelmente trouxe menores custos ao hospital. Além disso, possibilitou a redução do tempo de espera dos pacientes de ambulatórios e maior possibilidade de treinamento para os graduandos e especializandos na área de coloproctologia. Estas condições são bastante citadas na literatura, comprovando tendência para as operações anorretais ${ }^{1-3}$. Chamou-se atenção de que um estudo que analisasse estas variáveis fosse realizado no Hospital Universitário Cajuru, com suas conclusões possivelmente empregadas em mudanças na rotina do serviço.

A população estudada foi submetida a operações que seguem os padrões técnicos consagrados. Os dois grupos apresentaram homogeneidade nas suas características demográficas, de acordo com a análise estatística realizada, pois não houve diferença significativa entre os grupos nas variáveis sexo, idade e procedimento cirúrgico realizado. A diferença entre os dois grupos estudados foi apenas na técnica anestésica empregada, amplamente citadas na literatura como seguras e efetivas ${ }^{1}$.

Há constante preocupação da literatura médica sobre a seleção de pacientes que possam ser candidatos à realização de procedimentos anorretais ambulatoriais $^{11-12}$. Uma análise de 3725 pacientes operados em regime ambulatorial publicada em 2000 por Roche e Marti mostra que cerca de $70 \%$ dos pacientes puderam ser anestesiados com bloqueio local ${ }^{13}$. Os $30 \%$ restantes foram submetidos à raquianestesia. Neste trabalho, os autores evidenciam as melhores indicações para as operações anorretais ambulatoriais.
A inclusão de pacientes com risco anestésico pré-operatório ASA I e II foi respeitada conforme orientação da Sociedade Americana de Cirurgiões de Cólon e Reto $^{1}$, baseada em ampla revisão sobre o tema. Pacientes com estado físico ASA III e IV foram excluídos do estudo, por apresentarem maior risco de complicações clínicas.

Boa parte dos estudos comparando técnicas anestésicas diferentes para operações anorretais foca sua população em pacientes submetidos a hemorroidectomias ${ }^{9,14}$. Este estudo incluiu igualmente pacientes portadores de outras doenças anorretais benignas com indicações cirúrgicas, como fissuras anais, fístulas e condilomas. Há mínima possibilidade de viés neste aspecto, visto que o porte cirúrgico, os tempos operatórios e os materiais utilizados nestas operações são bastante equivalentes ${ }^{15}$. Infecções agudas perianais foram excluídas por se tratarem de procedimentos de emergência, não realizados de acordo com os requisitos técnicos deste estudo.

A divisão entre os grupos foi aleatória entre os pacientes. Devido a questões operacionais do Serviço de Anestesiologia do Hospital Universitário Cajuru, nem sempre o mesmo profissional estava disponível nas tardes em que os pacientes foram operados. Entretanto, foi mantida uma padronização técnica impecável entre os dois anestesiologistas que participaram do estudo.

Assim como a técnica anestésica, a técnica de bloqueio perianal local no grupo II e a técnica cirúrgica utilizada nos pacientes seguiram minuciosa sistemática. Todos os pacientes foram operados pelo mesmo cirurgião. Todos os bloqueios perianais locais nos pacientes do grupo II foram realizados igualmente pelo mesmo cirurgião, pela consagrada técnica de Schneider, de 1950. Até hoje, poucas modificações foram feitas nesta técnica de infiltração original, e esta é a maneira mais utilizada na literatura mundial para o bloqueio perianal local ${ }^{4}$.

Os achados comparativos entre os grupos relacionados ao tempo de procedimento anestésico-cirúrgico (medição indireta de tempo de ocupação da sala cirúrgica) mostraram diferença significativa. Houve redução do tempo de realização destes procedimentos nos pacientes do grupo II (tempo médio de 44,08 minutos), quando comparados ao grupo I (53,1 minutos), com $\mathrm{p}=0,0339$. Pode-se atribuir este fato a múltiplos motivos. Primeiramente, há necessidade de assepsia manual do anestesiologista para a realização das raquianestesias. Em segundo lugar, a técnica de 
raquianestesia pode ser demorada por dificuldades técnicas em determinados pacientes. Além disso, uma espera significativa de tempo para que o efeito do anestésico seja efetivado muitas vezes se faz necessária. Todos estes fatores, entre outros, podem justificar um maior tempo de realização de uma raquianestesia, visto que uma aplicação venosa de propofol claramente é mais rápida $^{2}$. Com estes achados, pode-se afirmar que o tempo de ocupação da sala cirúrgica é menor com a anestesia combinada. Indiretamente, uma sala de ambiente cirúrgico pode ter mais tempo disponível para a realização de outras operações, com a redução de sua ocupação, se os pacientes forem anestesiados conforme a técnica de anestesia combinada. Isto pode tornar a atividade cirúrgica diária mais ágil, e permite realização de maior número de operações num determinado período.

Resultados semelhantes foram encontrados por importantes estudos da literatura. Li et al., em estudo randomizado controlado publicado em 2000, compararam três grupos de pacientes submetidos a operações anorretais através de 3 tipos de anestesia: propofol venoso associado à anestesia local (grupo I); raquianestesia (grupo II) e anestesia geral (grupo III) ${ }^{2}$. Neste importante trabalho, uma das variáveis analisadas foi o tempo de realização da técnica de anestesia. Houve redução de cerca de $45 \%$ do tempo de anestesia nos pacientes submetidos à anestesia combinada do grupo I em relação aos demais grupos ${ }^{2}$. Sun et al., em 2006, publicaram estudo retrospectivo comparando a anestesia combinada (propofol e ketamina venosos associados ao bloqueio perianal local) com anestesia geral $^{3}$. Os achados de tempo médio de ocupação da sala cirúrgica foram de 27 minutos no grupo de anestesia combinada e 40 minutos no grupo de anestesia geral, com significância estatística ${ }^{3}$. Houve, portanto, importante redução do tempo de utilização do ambiente cirúrgico na anestesia combinada, achado compatível com o do presente estudo. Haveran et al., em análise de 180 pacientes submetidos à hemorroidectomia com bisturi harmônico com anestesia combinada (propofol com ketamina e local), demonstraram sucesso e aceitação do procedimento em 99,4\% dos $\operatorname{casos}^{9}$. O tempo médio de procedimento anestésico cirúrgico neste estudo foi de 28 minutos, demonstrando a agilidade deste tipo de anestesia para as referidas operações.

Este trabalho não analisou o grau de satisfação dos pacientes com as diferentes técnicas de anestesia. Entretanto, alguns estudos na literatura evidenciam que a maioria dos pacientes aprova o procedimento ambulatorial, principalmente com anestesia venosa associada ao bloqueio local, e repetiriam o procedimento outras vezes, se necessário fosse ${ }^{12,16}$. O benefício de não sentir dor durante a aplicação do anestésico local pela hipnose da anestesia venosa é a grande vantagem relatada deste método de anestesia combinada.

O grau de satisfação da equipe cirúrgica e anestésica, nos procedimentos com anestesia combinada, é ponto interessante deste presente estudo, apesar de subjetivo. A experiência pôde determinar uma excelente satisfação dos anestesiologistas por poder realizar procedimentos rápidos e efetivos sem maiores complicações. A satisfação da equipe cirúrgica também foi considerada alta, pelas condições intra-operatórias ideais. Notou-se maior satisfação da enfermagem e administração do ambiente cirúrgico por poder utilizar as salas operatórias por menos tempo, permitindo assim maior rotatividade de pacientes.

A anestesia venosa com propofol associada ao bloqueio perianal local é um método eficaz, conforme múltiplos relatos da literatura. Permite inclusive sedação dos pacientes em decúbito ventral, conforme a casuística de Read e colaboradores ${ }^{8}$.

As técnicas de raquianestesia e anestesia venosa com propofol associada ao bloqueio perianal local são igualmente consagradas na realização de operações anorretais, permitem boas condições operatórias e são utilizadas mundialmente. Entretanto, há nítida redução do tempo de ocupação de salas cirúrgicas nos pacientes operados com anestesia combinada.

\section{CONCLUSÕES}

As operações anorretais realizadas sob anestesia combinada (propofol venoso associado ao bloqueio perianal local) apresentaram menor tempo de ocupação de sala cirúrgica do que as operações realizadas sob raquianestesia, com significância estatística. As vantagens decorrentes deste fato podem trazer muitos benefícios aos hospitais e às equipes de cirurgiões e anestesiologistas, por criarem um fato importante: maior disponibilidade de tempo.

\section{AGRADECIMENTOS}

Agradecemos ao incansável auxílio dos médicos e residentes do Serviço de Anestesiologia do Hospital Universitário Cajuru (SAHUC) pelo irrestrito apoio para a realização deste estudo. 
ABSTRACT: Introduction: around ninety percent of anorectal surgical procedures are performed as day cases. The choice of a proper anesthetic technique is important to achieve reduced time in the operating rooms, hospital stay and low costs. There is no evidence in the literature that a superior type of anesthesia for these procedures exists. Objective: to compare the time spent on operating rooms in patients submitted to anorectal surgical procedures through spinal anesthesia (0,5\% bupivacaine) with combined anesthesia (propofol and local perineal block with $2 \%$ lidocaine and $0,5 \%$ bupivacaine). Methods: Fifty patients were operated with spinal anesthesia (group one) and forty-nine patients were operated with combined anesthesia (group two). The type of anorectal procedure and the time spent on anesthetic and surgical procedures were analyzed. Results: There were no significant differences between the studied groups regarding the type of operation, gender and age. The time of anesthetic and surgical procedures was 53,1 minutes in group one and 44,08 minutes in group two $(p=0,034)$. Conclusions: These anesthetic techniques had similar efficacy. There was a lower time of anesthetic and surgical procedures in the combined anesthesia group, with statistical significance.

Key words: Spinal Anesthesia; Propofol; Ambulatory Surgical Procedures; Anal Canal; Colorectal Surgery.

\section{REFERÊNCIAS}

1. Place R, Hyman N, Simmang C, Cataldo P, Church J, Cohen J, et al. Practice parameters for ambulatory anorectal surgery. Dis Colon Rectum. 2003; 46(5):573-6.

2. Li S, Coloma M, White PF, Watcha MF, Chiu JW, Li H, et al. Comparison of the costs and recovery profiles of three anesthetic techniques for ambulatory anorectal surgery. Anesthesiol. 2000; 93(5):1225-30.

3. Sun MY, Canete JJ, Friel JC, McDade J, Singla S, Paterson $\mathrm{CA}$, et al. Combination propofol/ ketamine is a safe and efficient anesthetic approach to anorectal surgery. Dis Colon Rectum. 2006; 49(7):1059-65.

4. Schneider H.C. Hyaluronidase with local anesthesia in anorectal surgery. Am J Surg. 1954;88(5): 703-6.

5. Johanson JF, Sonnenberg A. The prevalence of hemorrhoids and chronic constipation: an epidemiologic study. Gastroenterology 1990;98(2):380-6.

6. Bleday R, Pena JP, Rothemberger DA, Golberg SM, Buls JG. Symptomatic hemorroids: current incidence and complications of operative therapy. Dis Colon Rectum 1992;35(5):477-82.

7. Fleischer M, Marini CP, Statman R, Capella J, Shevde K . Local anesthesia is superior to spinal anesthesia for anorectal surgical procedures. Am Surg. 1994;60(11):812-5.

8. Read TE, Henry SE, Hovis RM, Fleshman JW, Birnbaum EH, Caushaj PF, et al. Prospective evaluation of anesthetic technique for anorectal surgery. Dis Colon Rectum. 2002;45(11):1553-60.

9. Haveran LA, Sturrock PR, Sun MY. Simple harmonic scalpel hemorrhoidectomy utilizing local anesthesia combined with intravenous sedation: a safe and rapid alternative to conventional hemorrhoidectomy. Int J Colorectal Dis. 2007;22(7):801-6.

10. Imbelloni LE, Vieira EM, Gouveia MA, Cordeiro JA. Raquianestesia posterior para cirurgias anorretais em regime ambulatorial: estudo piloto. Rev Bras Anestesiol. 2004;54(6):774-80.

11. Sobrado CW, Nahas SC, Marques CF, Habr-Gama A. Cirurgia ambulatorial sob anestesia local em proctologia: experiência e análise do resultado de 503 operações. Rev bras Coloproct. 2001; 21(4):228-33.

12. Henriques AC, Horta SHC, Pezzolo S, Waisberg J, Boratto SDF, Helal S, et al. Procedimentos cirúrgicos em pacientes proctológicos selecionados sob anestesia local: estudo de 150 casos. Arq Gastroenterol. 2000;37(3):158-61.

13. Roche B, Marti MC. Outpatient proctological surgery: an analysis of 3725 cases. Tech Coloproctol. 2000;4(1):35-8.

14. Lacerda Filho A, Melo JRC. Hemorroidectomia em regime ambulatorial sob anestesia local: estudo prospectivo de 50 casos. Rev bras Coloproct . 1995;15(4):206-10.

15. Smith LE. Ambulatory surgery for anorectal diseases: an update. South Med J. 1986; 79(2):163-6.

16. Thompson-Fawcett MW, Cook TA, Baigrie RJ, Mortensen NJM. What patients think of day-surgery proctology. Br J. Surg 1998;85(10):1388.

Endereço para correspondência:

PAULO GUSTAVO KOTZE

Rua Jaime Balão, 975 - ap. 106 - Curitiba - PR - CEP 80040-340

Fone/Fax: (41) 3022-5500

e-mail:pgkotze@hotmail.com 